\title{
$\checkmark$ Research Square \\ Defining the Sensitivity Landscape of 74,389 EGFR Variants to Tyrosine Kinase Inhibitors
}

\section{Lei An}

Henan University

\section{Shuqing Chen}

Typhoon HealthCare

\section{Guangyao Wu}

Henan University

Chang Liu

Henan University

\section{Zhenxing Wang}

Henan University

Chunli Wang

Henan University

\section{Zeyuan Shi}

Henan University

Chenguang Niu

Henan University

Xiaodong Li

Henan University

Wenxue Tang

Zhengzhou University

Hongen Xu ( $\nabla$ hongen_xu@zzu.edu.cn )

Zhengzhou University

\section{Yueqiang Wang}

Typhoon HealthCare

\section{Research}

Keywords: non-small-cell lung carcinoma, tyrosine kinase inhibitor, EGFR rare mutation, functional classification of variants, deep mutational scanning

Posted Date: September 1st, 2021

DOl: https://doi.org/10.21203/rs.3.rs-847350/v1 
License: (c) (i) This work is licensed under a Creative Commons Attribution 4.0 International License. Read Full License 


\section{Abstract}

Background: Tyrosine kinase inhibitors (TKIs) therapy is a standard treatment for patients with advanced non-small-cell lung carcinoma (NSCLC) when activating epidermal growth factor receptor (EGFR) mutations are detected. However, except for the well-studied EGFR mutations, most EGFR mutations lack treatment regimens.

Methods: We constructed two EGFR variant libraries containing substitutions, deletions, or insertions using the saturation mutagenesis method. All the variants were located in the EGFR mutation hotspot (exons 18-21). The sensitivity of these variants to afatinib, erlotinib, gefitinib, icotinib, and osimertinib was systematically studied by determining their enrichment in massively parallel cytotoxicity assays using an endogenous EGFR-depleted cell line, PC9.

Results: A total of 3,914 and 70,475 variants were detected in the constructed EGFR Substitution-Deletion (Sub-Del) and exon 20 Insertion (Ins) libraries, accounting for $99.3 \%$ and $55.8 \%$ of the designed variants, respectively. Of the 3,914 Sub-Del variants, 813 were highly enriched in the reversible TKI (erlotinib, gefitinib, icotinib) cytotoxicity assays and 51 were enriched in the irreversible TKI (afatinib, osimertinib) cytotoxicity assays. For the 70,475 Ins variants, insertions at amino acid positions $770-774$ were highly enriched in all the five TKI cytotoxicity assays. Moreover, the top $5 \%$ of the enriched insertion variants included a glycine or serine insertion at high frequency.

Conclusions: We present a comprehensive reference for the sensitivity of EGFR variants to five commonly used TKIs. The approach used here should be applicable to other genes and targeted drugs.

\section{Significance Statement}

Mutation-directed cancer precision medicine requires pre-interpretation of variants response on targeted drugs. For Asian lung adenocarcinoma patients, epidermal growth factor receptor (EGFR) is a commonly mutated gene with many rare mutations that lack drug response interpretations. We conducted a systematic cytotoxicity screening of EGFR mutations on five tyrosine kinase inhibitors. We found that patients with rare EGFR mutations are most likely to benefit from osimertinib therapy compared to afatinib, erlotinib, gefitinib, or icotinib therapy. This study provides the first case of deep mutational scanning that simultaneously assayed substitution, deletion, and insertion variants. This approach is applicable for other oncogenes and targeted drugs.

\section{Background}

The incidence of epidermal growth factor receptor (EGFR) mutations is reported to be $28.6 \%$ among populations with non-small-cell lung carcinoma (NSCLC). East Asian and Southeast Asian populations have a higher incidence (41.3-57.2\%) than the European population (8.0-20.2\%) [1]. Clinical trials have shown that EGFR-tyrosine kinase inhibitor (EGFR-TKI) treatment of patients with NSCLC is superior to chemotherapy in terms of progression-free survival and serious adverse effects [2, 3]. Several EGFR-TKIs 
(for example, afatinib, erlotinib, gefitinib, and osimertinib) have been approved for first-line treatment of patients with advanced NSCLC having activating EGFR mutations [4]. Usually, an EGFR mutation testing is performed to check the pattern of mutations in patients, which enables the physicians to determine whether, and which, EGFR-TKI could be used. Statistical analysis of EGFR mutation records in the Catalogue of Somatic Mutations in Cancer (COSMIC) database shows that, apart from the secondary mutations (for example, p.Thr790Met, p.Cys797Ser), p.Leu858Arg (41\%) and exon 19 deletions (47\%) together account for $88 \%$ of the mutations in all the records, whereas rare mutations account for about $12 \%$ of all mutations [5]. The rare EGFR mutations mainly comprise missense variants ( $>63 \%$ ) and exon 20 insertions ( 17\%) [5]. Although rare EGFR mutations are reportedly less prevalent, the high incidence of NSCLC has increasingly led to their detection in the clinic [6], making it impossible to ignore them. Unlike a limited number of high-prevalence EGFR mutations, TKI sensitivity for rare mutations and a large number of potential mutations has not been systematically studied.

Several groups have tried to evaluate the clinical relevance of EGFR variants on a large scale. One group developed a mixed-all-nominated-mutants-in-one (MANO) method and applied it to 101 nonsynonymous EGFR variants [7]. The process of the MANO method is labor intensive, which makes it challenging to evaluate the functions of variants on a large scale, for example, for several thousands of variants. Another group reported screening for activating EGFR mutations using a library of 7,216 randomly mutated single-nucleotide variants [8]. This variant library was generated by the error-prone PCR method, which can barely cover all the interested variants.

In view of the lack of economical and efficient methods, it is highly challenging to systematically study the TKI sensitivity of rare EGFR mutations on a large scale. Deep mutational scanning (DMS) is a cuttingedge technology that enables the functional analysis of large numbers of variants simultaneously $[9,10]$. Usually, a mutant library is first constructed employing synthetic biology or genome editing methods [11, 12], and functional impacts of variants are determined through parallel functional assays. To date, variants of a few clinically actionable genes, such as BRCA1, PPARG, TP53, PTEN, TPMT, NUDT15, SCN5A, CYP2C9, CYP2C19, CXCR4, CCR5, ADRB2, and MSH2 [12-24], have been extensively studied using the DMS method, highlighting its potential for assessing the TKI sensitivity of rare and potential EGFR mutations. In this study, based on the DMS method, we developed massively parallel cytotoxicity assays and systematically determined the sensitivity landscape of the variants to five EGFR-TKIs (Fig. 1).

\section{Materials And Methods}

\section{Cells Lines and Reagents}

HEK 293 and A549 cell lines cryopreserved in our laboratory were used. PC9 cells were obtained from Beijing Cancer Hospital and authenticated via short tandem repeat (STR) profiling. All cells were tested to be mycoplasma negative. The genetic background of PC9 cells was further checked by next-generation sequencing using the Illumina TruSight Cancer panel. Cells were maintained according to the instructions prescribed by American Type Culture Collection (ATCC). Unless otherwise noted, all cell culture reagents 
were purchased from Thermo Fisher Scientific (USA), and all molecular cloning reagents were purchased from New England Biolabs (NEB, USA). Afatinib (S1011), erlotinib (S1023), icotinib (S2922), and osimertinib (S7297) were purchased from Selleck Chemicals and gefitinib (SML1657) was purchased from Sigma-Aldrich. Total EGF Receptor (10001-R021) and anti-EGFR-PE (352904) antibodies were purchased from Sino Biological (China) and BioLegend (USA), respectively.

\section{CRISPR/Cas9 Single Guide RNA (sgRNA) for Endogenous EGFR Deletion}

Ten EGFR targeting sgRNAs were designed using the online tool, CHOPCHOP (https://chopchop.cbu.uib.no/). The sgRNAs cloning were performed according to the reported protocol [25]. CRISPR/Cas 9 lentiviral particles were separately produced and then introduced into A549 cells. The gene-editing efficiency of the sgRNAs was determined using fluorescent-activated cells sorting (FACS) analysis and immunoblotting.

\section{Construction of Saturation Mutagenesis Libraries}

EGFR saturation mutagenesis libraries were constructed based on the Mutagenesis by Integrated TilEs (MITE) method reported previously [11], with necessary modifications. The complete SubstitutionDeletion (Sub-Del) Library was obtained by mixing six sub-libraries at equal mass ratios. The complete Insertion (Ins) Library was obtained by mixing three sub-libraries (Ins-1, Ins-2, Ins-3) in the mass ratio of 1:5:5. The completeness and uniformity of the variant libraries were verified through next-generation sequencing using a HiSeq sequencer (Illumina).

\section{Lentivirus Production}

Lentivirus was produced and titered as reported previously [15], with minor modifications. For lentivirus production, five million 293T cells were pre-seeded on $10 \mathrm{~cm}$ dishes, the first day evening. Cells were cotransfected with the variant library plasmid and two helper plasmids (psPAX2 and pMD2.G) at a molar ratio of 1:1:1 using a cationic polymer transfection reagent, EZ Trans (LIFE iLab, China), according to the manufacturer's instructions. The medium was replaced $24 \mathrm{~h}$ later and the virus particles were collected and filtered through $0.45 \mu \mathrm{m}$ filters (Millipore), 24 or $48 \mathrm{~h}$ later.

\section{TKI Cytotoxicity Screening}

PC9 cells were transduced with a Cas 9 expression lentivirus and selected on puromycin for 2 weeks. PC9 cells stably expressing Cas9 (PC9-Cas9) were cultured in large quantities and seeded in twenty $15 \mathrm{~cm}$ dishes ( 15 million cells per dish), the first day evening. Subsequently, these cells were transduced with a pooled virus of the Sub-Del/Ins library (10 dishes for each library) at a multiplicity of infection of $\sim 0.25$. Transduced cells were selected on a medium with puromycin and blasticidin for 2 weeks. For the TKI sensitivity screening, cells transduced with the Sub-Del/Ins library were separately seeded in eight $15 \mathrm{~cm}$ dishes ( 15 million cells per dish) and treated with different TKIs or dimethyl sulfoxide (DMSO): two for afatinib (50 nM/600 nM), one for erlotinib (600 nM), one for gefitinib (600 nM), one for icotinib (600 nM), two for osimertinib (200 nM/600 nM), and one for DMSO (control). The remaining cells ( $>5$ million cells 
for each library) were collected as the time 0 samples. Cells were trypsinized, manually counted, and reseeded every 2 days for 14 days. Dishes of proper size were used each time to ensure that the cell density was maintained within a proper range. The TKI assays were independently repeated once (Additional file 1: Figure S1). After 14 days of TKI treatment (time 1), cells were harvested for genomic DNA (gDNA) extraction.

\section{Amplification and Sequencing of Mutational Regions}

The first-round PCR was performed to amplify a 1.1 kb DNA fragment covering the entire EGFR mutational region from the gDNA. Multiple $50 \mu \mathrm{L}$ PCRs were performed for each sample. PCR products from each sample were separately pooled and purified with a Universal DNA Purification Kit (Tiangen, China). In the second-round PCR, the Sub-Del library mutational region was amplified with corresponding tagged primers (Additional file 10: Table S1) using the $1.1 \mathrm{~kb}$ fragments as templates. In contrast, the Ins library mutational region was amplified from the corresponding templates. All PCRs were performed using the Q5 High-Fidelity DNA Polymerase (NEB). The amplicons were purified using a Universal DNA Purification Kit. Next-generation sequencing libraries were constructed according to the manufacturer's protocol (VAHTS, China). All samples were sequenced on the HiSeq platform (Illumina) using a $2 \times 150$ paired-end configuration.

\section{Data Processing}

Specifically, raw overlapping paired reads (read1 and read2) were merged into single reads using the bbmerge tool with default parameters [26]. For Sub-Del libraries, each read was assigned to one reference sequence using the USEARCH tool with parameters of -usearch_global -strand both -id 0.95 [27]. For Ins libraries, each read was assigned to one reference sequence with the same USEARCH tool but with a different value of id parameter (0.90). The successfully assigned reads in Sub-Del and Ins libraries were translated into AA and were subjected to diamond blastp analysis against the reference protein sequences to call variants [28]. The frequency of each mutation was calculated as the ratio of the variant count to the count of valid variants. Thereafter, the frequency of each variant was weighted with the ratio of cell count change relative to time 0 . To assess the enrichment or depletion of each variant, we calculated the log2-fold change in variant frequency relative to the time 0 samples. Heatmap and violin plots were created using the R pheatmap and ggplot2 packages, respectively.

\section{Results}

\section{Construction of 74,389 EGFR Variants}

EGFR pathogenic variants, including substitutions, deletions, and exon 20 insertions [29], are mainly located in exons 18-21 (residues 688-875). To cover all the major types of mutations, two EGFR libraries were constructed using the MITE method [11]. Parallel synthesized oligonucleotides were used to construct a saturation EGFR Sub-Del library containing 3,572 (theoretical number) single AA substitution (Sub) variants, 188 (theoretical number) single AA deletion (Del-1) variants, and 182 (theoretical number, 
for cloning reason, six deletion variants were not covered) two consecutive AA deletion (Del-2) variants. All Sub-Del library variants were located at AA positions 688-875. Similarly, multiple degenerate primers were used to construct a saturation EGFR Ins library containing 126,300 (theoretical number) in-frame insertion variants. All Ins library variants were located in the EGFR insertion hotspot (AA position, 762776) and consisted of one, two, or three tandem NNS codons ( $N$ = adenine, cytosine, guanine, or thymine; $\mathrm{S}=$ cytosine or guanine) inserted between adjacent $\mathrm{AAs}$, with each variant containing one insertion in each of the AA positions. The Ins library was composed of three subtype libraries: Ins-1 library (300 variants, each with a single-AA inserted), Ins-2 library (6,000 variants, each with a double-AA inserted), and Ins-3 library (120,000 variants, each with a triple-AA inserted). Variants of Sub-Del and Ins libraries were cloned into lentiviral vectors, and the uniformity and completeness of each library were determined by high-throughput sequencing of the plasmids. The Sub-Del library showed decent uniformity (Additional file 2: Figure S2a), and $99.3 \%$ of the designed variants were detected more than 20 times (>20 reads) (Additional file 3: Figure S3a). Similarly, variants of the Ins library also showed high uniformity (Additional file 2: Figure S2b). Because the Ins library was derived from mixing the Ins-1, Ins-2, and Ins-3 libraries at a mass ratio of 1:5:5, the ratio of the average proportion of each variant from Ins-1, Ins-2, and Ins-3 libraries was 80:20:1. Not surprisingly, most of the designed variants in the Ins-1 (99.7\%) and Ins-2 $(93.6 \%)$ libraries were detected (>20 reads for each variant). However, only $53.8 \%$ of the designed Ins -3 library variants were detected ( $>20$ reads for each variant) (Additional file 3: Figure S3b). Overall, the coverage and uniformity of Sub-Del and Ins libraries were as expected, and the total number of detected variants was 74,389 .

\section{Deletion of Endogenous EGFR in the PC-9 Cell Model}

The human lung adenocarcinoma cell line, PC9 (contains EGFR exon 19 deletions), was chosen as the model. The cell line identity was confirmed by STR profiling and genomic mutations were further reviewed through next-generation sequencing. A Glu746-Ala750 deletion was confirmed in EGFR exon 19 and a p.Arg248GIn mutation was detected in TP53. The p.Arg248GIn variant is a gain-of-function mutation that promotes tumorigenesis [30]. These results indicate that the PC9 cell line is a genetically clear cell model for evaluating the sensitivity of EGFR variants to TKIs.

To eliminate the endogenous EGFR, we separately introduced Cas9 and the sgRNA-EGFR variant (codonoptimized) into PC9 cells using two lentiviral vectors. Firstly, Cas9 was introduced into PC9 cells to obtain the PC9-Cas9 cell line, stably expressing Cas9. Subsequently, the sgRNA-EGFR variant was introduced into the PC9-Cas9 cells. The endogenous EGFR was knocked out using the CRISPR/Cas9 system and replaced with the exogenous EGFR variant in approximately 10 days. Because direct knockout of EGFR may seriously affect the viability of PC9 cells [31], we tested the CRISPR/Cas9 gene-editing efficiency in A549 cells (with a wildtype EGFR and mutated KRAS background). Considering that KRAS locates downstream of the EGFR signaling pathway, EGFR can be knocked out without seriously affecting the viability of A549 cells. FACS and western blotting analyses confirmed that the endogenous EGFR had been efficiently knocked out by CRISPR/Cas9 (Additional file 4-5, 11: Figure S4-S5, Table S2). We 
predicted that these validated CRISPR/Cas9 sgRNAs would also efficiently knockout the endogenous EGFR in PC9-Cas9 cells.

\section{EGFR-TKI Cytotoxicity Screening}

To systematically evaluate the sensitivity of EGFR variants to different TKIs, we separately introduced Sub-Del and Ins variants into the PC9-Cas9 cells and treated them with TKIs. Expression of exogenous EGFR variants was checked through FACS analysis and found to be slightly higher than that of EGFR in PC9 cells (Additional file 6: Figure S6). The concentrations of EGFR-TKIs were set mainly referring to the clinical plasma concentration of each TKI (Additional file 12: Table S3). Specifically, the concentrations of reversible TKIs (erlotinib, gefitinib, and icotinib) were set to $600 \mathrm{nM}$, whereas those of irreversible TKls were set at two levels. The afatinib clinical plasma concentration is much lower than $600 \mathrm{nM}$ [32]; we designed two assays for 50 and $600 \mathrm{nM}$ afatinib. Because incubation with $600 \mathrm{nM}$ osimertinib showed strong cytotoxicity to cells with Sub-Del variants in vitro, we designed two assays for 200 and $600 \mathrm{nM}$ osimertinib. The TKI treatments lasted 2 weeks and cells were counted every 2 days. The number of cells containing the Sub-Del variants in the erlotinib, gefitinib, icotinib, afatinib (50 nM), and osimertinib (200 $\mathrm{nM}$ ) assays decreased substantially at days 5 and 7, reached the minimum at day 9 , and then began to increase. The cell numbers in the afatinib (600 nM) and osimertinib (600 nM) assays reached the minimum at days 11 and 15, respectively (Fig. 2a). For cells containing the Ins variants, the minimum cell number in the afatinib $(600 \mathrm{nM})$ and osimertinib $(600 \mathrm{nM})$ assays was reached at days 9 and 13, respectively, whereas the cell number in other TKI assays was not decreased during 2-week of TKI treatment (Fig. 2b).

\section{Osimertinib Inhibited Almost all Types of Variants}

To investigate the enrichment of variants after incubation with TKIs, we harvested cells from each TKI assay for gDNA extraction, mutation region amplification, and high-throughput sequencing. The relative drug sensitivity for each variant was defined as the fold change of a variant's theoretical cell number post-TKI screening (time 1 ) to that at the early time point (time 0 ). The theoretical cell number for each variant was calculated using the read proportion of a variant among the total valid reads multiplied by the final cell number in each assay. Compared with reversible TKIs (erlotinib, gefitinib, and icotinib), irreversible TKIs (afatinib $50 \mathrm{nM}$ and osimertinib $200 \mathrm{nM}$ ) showed stronger inhibition for Sub-Del variants (enrichment score mean: afatinib, 0.331; erlotinib, 0.793; gefitinib, 1.73; icotinib, 4.44; osimertinib, 0.388; false discovery rate (FDR)-adjusted $P$ values <0.0001) (Fig. 2c). For the Ins variants, osimertinib (200 nM) had a greater inhibition than the other TKIs (enrichment score mean: afatinib, 113; erlotinib, 89.3; gefitinib, 111; icotinib, 176; osimertinib, 4.46; FDR-adjusted P values <0.0001) (Fig. 2d). Overall, these results indicated that the Ins library variants were more resistant to TKI treatment than the Sub-Del library variants, and osimertinib (200 $\mathrm{nM})$ showed stronger inhibition for variants in both the libraries.

To investigate whether distinct EGFR variant subtypes present different sensitivity against the same EGFR-TKI, we separately analyzed relative drug sensitivity of the Sub, Del-1, and Del-2 variants in the SubDel library and the Ins-1, Ins-2, and Ins-3 variants in the Ins library. No significant difference in the 
inhibition of Sub, Del-1, and Del-2 variants to any of the five TKIs was observed (Fig. 2e, FDR-adjusted P values $>0.05$ ). In contrast, the sensitivity of Ins-1, Ins-2, and Ins-3 variants to each of the five TKIs was significantly different (Fig. 2f, FDR-adjusted $P$ values $<0.05$ ). However, the difference in enrichment scores ( $\log 2)$ cannot be entirely attributed to TKI treatment because differences were also observed for Ins-1, Ins-2, and Ins-3 variants in TKI-free assay (control).

\section{Cytotoxicity Screening Results were Consistent with Known Clinical Annotations}

Next, we evaluated the consistency of TKI screening results with known clinical annotations. For this, we compiled a list of EGFR mutations from the Clinical Interpretation of Variants in Cancer (CIViC) knowledgebase and compared the clinical annotations (resistant or sensitive) of mutations with the enrichment scores ( $\log 2)$ obtained in cytotoxicity assays by plotting those mutations on a scale bar comprising all the results (Additional file 7: Figure S7). TKI-sensitive variants (such as p.Leu858Arg and p.Gly719Ala/Ser) were located in the middle or lower half of the bar, whereas TKI-resistant variants (such as p.Thr790Met) were located in the middle or upper half of the bar (Additional file 7: Figure 7a). Similarly, the annotated Ins variants were distributed at expected positions in the bars (Additional file 7: Figure 7b). However, some annotated variants, such as p.Cys797Ser (0.53) and p.Ser768lle (1.19), had contradictory enrichment scores in the osimertinib assay. These discrepancies could be attributed to the presence of additional mutations. For example, EGFR mutation combinations in cis (on the same allele) or trans (on different alleles) might lead to altered drug response outcomes compared with those for independent mutations [33-36]. Indeed, p.Cys797Ser has been detected as a secondary mutation coexisting with p.Thr790Met in cis and results in resistance to osimertinib [33-35]. In addition, another variant, p.Gly724Ser, has been associated with increased sensitivity to osimertinib when combined in trans with p.Leu858Arg than with exon 19 deletions [36]. In general, our TKI sensitivity evaluation results are consistent with the known annotation.

\section{Cytotoxicity Screening Identifies Potentially Drug Resistant Variants}

The variant enrichment scores ( $\log 2)$ in each TKI assay for each variant were presented as heatmaps (Fig. 3, 4, Additional file 8-9: Figure S8-S9). For Sub-Del variants, 280 (7.2\%) variants in afatinib assay (50 $\mathrm{nM}), 841$ (21.5\%) variants in erlotinib assay, 1,802 (46.2\%) variants in gefitinib assay, 3,440 (88.0\%) variants in icotinib assay, and $271(7.2 \%)$ variants in osimertinib assay $(200 \mathrm{nM})$ of all variants had a positive enrichment score $(\log 2)$, indicating that the number of those variants were increased after 2 weeks of TKI incubation. We divided all substitution variants into two categories, the complex and simple variants. The complex variants can only be achieved through two or three point mutations in the corresponding codon whereas the simple variants can be obtained through one point mutation. Under normal circumstances, the probability of multiple independent point mutations occurring in the same codon is relatively low, and therefore, simple variants are more likely to be encountered in the clinic. Enriched variants from all three reversible TKI assays have 813 variants (complex variants $=571$, simple variants $=242$ ) in common (Fig. $5 \mathrm{a}, \mathrm{c}, \mathrm{e}$ ). The variants enriched in the two irreversible TKI assays have 51 variants (complex variants $=36$, simple variants $=15$ ) in common (Fig. 5 b, d, f). Overall, in our 
systematic cytotoxicity screening, we found 813 and 51 substitution variants that potentially mediate resistance to EGFR reversible and irreversible TKIs, respectively.

\section{The Top 5\% Enriched Insertions Showed Insertional Position and AA Type Preference}

For Ins variants, the proportion of variants with positive enrichment scores (log2) was much larger than of their Sub-Del counterparts: $95 \%$ for afatinib (50 nM) assay, 93\% for erlotinib assay, $94 \%$ for gefitinib assay, $97 \%$ for icotinib assay, and $57 \%$ for osimertinib (200 nM) assay. To study whether the insertional position and the AA type affect the sensitivity of exon 20 insertions to different TKIs, we selected the top $5 \%$ enriched insertions from each TKI assay and determined the cumulative frequency of the inserted AA at each AA position. The top enriched exon 20 insertions shared many common features (Fig. 6 a-f): (1) the highly enriched insertion variants showed preference for insertion at AA positions $770-774$; $(2)$ the highly enriched insertion variants showed preference of glycine and serine. To evaluate the sensitivity of the clinically detected exon 20 insertions to TKIs, we determined the cumulative frequency of insertional AA in variants from the Chinese population [37] and COSMIC database (Fig. $6 \mathrm{~g}-\mathrm{i}$ ). The detected rare exon 20 insertions were mainly concentrated within AA positions 770-774. Our results explain the resistance of these clinically identified exon 20 insertions to TKIs. Together, these results reveal that insertion in the EGFR AA positions 770-774 might induce a stronger resistance to TKIs, especially when the insertions contain glycine or serine.

\section{Discussion}

In the post-genome era, a massive number of variants of unknown significance (VUS) has been identified by high-throughput sequencing; however, it is still a big challenge for functional interpretation of VUS in the clinical context. To study the TKI sensitivity of rare EGFR mutations, we designed cytotoxicity assays and systematically evaluated the sensitivity of 74,389 EGFR variants to five commonly used EGFR-TKIs. Afatinib and osimertinib showed intense and relatively persistent inhibition of all types of EGFR variants (substitutions, deletions, and exon 20 insertions) among the five tested EGFR-TKIs. Considering the tolerance to the treatment, the clinical plasma drug concentration of afatinib cannot reach $600 \mathrm{nM}$ [38], whereas for osimertinib, it can be more than $600 \mathrm{nM}$ [39]. Accordingly, we speculate that otherwise for additional evidence, patients with NSCLC having rare EGFR mutations (including exon 20 insertions) are more likely to benefit from osimertinib than from the other four TKIs. Compared with Sub-Del mutations, EGFR exon 20 insertion mutations were more difficult to deal with. A recent clinical study showed that patients with EGFR exon 20 insertions could benefit from high-dose (160 mg daily) osimertinib treatment [40]. With the combined use of synthetic biology and CRISPR/Cas9 methods, we can economically generate a large number of cell models with designed mutations of a specific gene. These cell models can partially simulate clinical samples and enable us to obtain rapid screening results through appropriate functional assays.

The systematic cytotoxicity screening of Sub-Del variants has led us to identify several potentially drugresistant substitution variants. We were curious whether these variants occurred with equal frequency in 
the clinic. Further analysis revealed that the substitution variants could be divided into complex and simple variants. The majority of EGFR substitution mutations detected in the clinic are simple variants and most of the complex variants are rarely detected. The difficulty in the mutation process partially explains why the highly enriched complex variants are rarely detected in the clinic [15]. To the best of our knowledge, we have designated substitution variants into complex and simple variants for the first time. More efforts should be invested on the functional interpretation of the simple variants in the clinical context.

The systematic screening revealed that EGFR exon 20 insertions at AA positions 770-774 would induce stronger TKI resistance, especially when the insertions contain glycine or serine. Clinically, excluding the high prevalence exon 20 insertions of p.Ala767_Val769dup and p.Ser768_Asp770dup, a large number of low prevalence exon 20 insertions are mostly concentrated at AA positions 770-774 [37, 41]. According to the theory of Vyse et al., EGFR exon 20 insertions in the loop region (AA positions 767-775) have altered three-dimensional structures and will stabilize the mutated EGFR in the active state even without ligand binding [41]. Our systematic screening results provided important experimental evidence supporting this theory.

The cytotoxicity assay-based method for functional interpretation of variants used in this study can also be applied to other genes and targeted drugs. At least 52 small molecule kinase inhibitors have been approved by US food and drug administration for targeted therapy [42]. An extensive functional interpretation of variants will enable us to clarify the targeted inhibitors applicable for the corresponding mutations. Moreover, high throughput sequencing of circulating tumor DNA allows us to forecast the potential drug-resistance events [43-46]. This will dramatically promote the development of precision medicine. Moreover, for newly developed small molecule targeted inhibitors, a pre-drug sensitivity screening at the laboratory stage will help forecast the suitability of drug candidates for patients with specific mutations, thereby drastically reducing the cost incurred at clinical trial stages.

Despite the significance of this work, there are several important caveats: (1) We only used PC9 cells for cytotoxicity screening. The genetic background of this cell line might have had an unwanted influence on the functional interpretation of variants $[12,19]$. The use of multiple NSCLC cell lines for parallel functional screening can significantly reduce such interference; (2) The expression of the exogenous EGFR variants was higher than that of endogenous EGFR in PC9 cells, which may lead to an overestimation of the TKI resistance of specific variants; (3) The endogenous EGFR of PC9 cells was deleted by CRISPR/Cas9. In theory, each of the engineered PC9 cells has one copy of the exogenous EGFR variant. However, several studies have demonstrated that EGFR variant combinations can significantly influence sensitivity to a specific TKI [33-36, 47]. Further studies are needed to uncover the TKI inhibition against combinations of EGFR variants.

\section{Conclusion}


In conclusion, systematic cytotoxicity screening revealed that patients with NSCLC having rare EGFR mutations are most likely to benefit from osimertinib treatment. We expect variants of other genes to be classified to assess their sensitivity to corresponding targeted inhibitors.

\section{Abbreviations}

AA: Amino acid; Afa: Afatinib; ATCC: American Type Culture Collection; CIViC: Clinical Interpretation of Variants in Cancer; COSMIC: Catalogue of Somatic Mutations in Cancer; DMS: Deep mutational scanning; DMSO: Dimethyl sulfoxide; EGFR: Epidermal growth factor receptor; EGFR-TKI: EGFR-tyrosine kinase inhibitor; Erl: Erlotinib; FACS: Fluorescent-activated cells sorting; FDR: False discovery rate; gDNA: Genomic DNA; Gef: Gefitinib; Ico: Icotinib; Ins: Insertion; MANO: Mixed-all-nominated-mutants-in-one; MITE: Mutagenesis by Integrated TilEs; NEB: New England Biolabs; NSCLC: Non-small-cell lung carcinoma; Osi: Osimertinib; sgRNA: Single guide RNA; STR: Short tandem repeat; Sub-Del: SubstitutionDeletion; TKI: Tyrosine kinase inhibitor; VUS: Variants of unknown significance.

\section{Declarations}

\section{Ethical Approval and Consent to participate}

Not applicable.

\section{Consent for publication}

All authors give consent for the publication of the manuscript in Journal of Experimental \& Clinical Cancer Research.

\section{Availability of data and materials}

The datasets used and analyzed during the current study are available from the corresponding author on reasonable request.

\section{Competing interests}

The authors declare no conflict of interest.

\section{Funding}

This work was supported by Henan Province Key Research and Promotion Project (202102310403, 192102310034) and Henan Province Research Programs of Medical Science and Technology (LHGJ20190544).

\section{Authors' contributions}


Y.W. designed research; L.A., S.C., G.W., C.L., Z.W., C.W., and Z.S. performed research; C.N., X.L., and W.T. contributed new reagents/analytic tools; H.X., and Y.W. analyzed data; and Y.W., H.X., and L.A. Wrote the paper.

\section{Acknowledgements}

We thank Dr. Kang Bin from Cancer Hospital of Peking University for PC9 cell line and Luo Yuqi for experimental assistance. We thank Dr. Ren Yanfang of the University of Rochester for his comments on the manuscript. The data analysis was supported by the Supercomputing Center at Zhengzhou University (Zhengzhou).

\section{References}

1. Soraas L, Stebbing J. Geographic Variation in EGFR Mutation Frequency in Lung Adenocarcinoma May Be Explained by Interethnic Genetic Variation. J Thorac Oncol. 2018, 13(3):454-458.

2. Kuan FC, Kuo LT, Chen MC, Yang CT, Shi CS, Teng D, Lee KD. Overall survival benefits of first-line EGFR tyrosine kinase inhibitors in EGFR-mutated non-small-cell lung cancers: a systematic review and meta-analysis. Br J Cancer. 2015, 113(10):1519-1528.

3. Wang S, Cang S, Liu D. Third-generation inhibitors targeting EGFR T790M mutation in advanced nonsmall cell lung cancer. J Hematol Oncol. 2016, 9:34.

4. Zhao Y, Liu J, Cai X, Pan Z, Yin W, Chen H, et al. Efficacy and safety of first line treatments for patients with advanced epidermal growth factor receptor mutated, non-small cell lung cancer: systematic review and network meta-analysis. BMJ 2019, 367:15460.

5. Harrison PT, Vyse S, Huang PH. Rare epidermal growth factor receptor (EGFR) mutations in nonsmall cell lung cancer. Semin Cancer Biol. 2020, 61:167-179.

6. Bray F, Ferlay J, Soerjomataram I, Siegel RL, Torre LA, Jemal A. Global cancer statistics 2018 : GLOBOCAN estimates of incidence and mortality worldwide for 36 cancers in 185 countries. CA Cancer J Clin. 2018, 68(6):394-424.

7. Kohsaka S, Nagano M, Ueno T, Suehara Y, Hayashi T, Shimada N, et al. A method of high-throughput functional evaluation of EGFR gene variants of unknown significance in cancer. Sci Transl Med. 2017, 9(416):eaan6566.

8. Chakroborty D, Kurppa KJ, Paatero I, Ojala VK, Koivu M, Tamirat MZ, et al. An unbiased in vitro screen for activating epidermal growth factor receptor mutations. J Biol Chem. 2019, 294(24):9377-9389.

9. Araya CL, Fowler DM. Deep mutational scanning: assessing protein function on a massive scale. Trends Biotechnol. 2011, 29(9):435-442.

10. Fowler DM, Stephany JJ, Fields S. Measuring the activity of protein variants on a large scale using deep mutational scanning. Nat Protoc. 2014, 9(9):2267-2284.

11. Melnikov A, Rogov P, Wang L, Gnirke A, Mikkelsen TS. Comprehensive mutational scanning of a kinase in vivo reveals substrate-dependent fitness landscapes. Nucleic Acids Res. 2014, 42(14):e112. 
12. Findlay GM, Daza RM, Martin B, Zhang MD, Leith AP, Gasperini M, et al. Accurate classification of BRCA1 variants with saturation genome editing. Nature. 2018, 562(7726):217-222.

13. Starita LM, Young DL, Islam M, Kitzman JO, Gullingsrud J, Hause RJ, et al. Massively Parallel Functional Analysis of BRCA1 RING Domain Variants. Genetics. 2015, 200(2):413-422.

14. Majithia AR, Tsuda B, Agostini M, Gnanapradeepan K, Rice R, Peloso G, et al. Prospective functional classification of all possible missense variants in PPARG. Nat Genet. 2016, 48(12):1570-1575.

15. Giacomelli AO, Yang X, Lintner RE, McFarland JM, Duby M, Kim J, et al. Mutational processes shape the landscape of TP53 mutations in human cancer. Nat Genet. 2018, 50(10):1381-1387.

16. Kotler E, Shani O, Goldfeld G, Lotan-Pompan M, Tarcic O, Gershoni A, et al. A Systematic p53 Mutation Library Links Differential Functional Impact to Cancer Mutation Pattern and Evolutionary Conservation. Mol Cell. 2018, 71(5):873.

17. Matreyek KA, Starita LM, Stephany JJ, Martin B, Chiasson MA, Gray VE, et al. Multiplex assessment of protein variant abundance by massively parallel sequencing. Nat Genet. 2018, 50(6):874-882.

18. Mighell TL, Thacker S, Fombonne E, Eng C, O'Roak BJ. An Integrated Deep-Mutational-Scanning Approach Provides Clinical Insights on PTEN Genotype-Phenotype Relationships. Am J Hum Genet. 2020, 106(6):818-829.

19. Suiter CC, Moriyama T, Matreyek KA, Yang W, Scaletti ER, Nishii R, et al. Massively parallel variant characterization identifies NUDT15 alleles associated with thiopurine toxicity. Proc Natl Acad Sci U S A. 2020, 117(10):5394-5401.

20. Glazer AM, Kroncke BM, Matreyek KA, Yang T, Wada Y, Shields T, et al. Deep Mutational Scan of an SCN5A Voltage Sensor. Circ Genom Precis Med. 2020, 13(1):e002786.

21. Zhang L, Sarangi V, Moon I, Yu J, Liu D, Devarajan S, et al. CYP2C9 and CYP2C19: Deep Mutational Scanning and Functional Characterization of Genomic Missense Variants. Clin Transl Sci. 2020, 13(4):727-742.

22. Heredia JD, Park J, Brubaker RJ, Szymanski SK, Gill KS, Procko E. Mapping Interaction Sites on Human Chemokine Receptors by Deep Mutational Scanning. J Immunol. 2018, 200(11):3825-3839.

23. Jones EM, Lubock NB, Venkatakrishnan AJ, Wang J, Tseng AM, Paggi JM, et al. Structural and functional characterization of $\mathrm{G}$ protein-coupled receptors with deep mutational scanning. Elife. 2020, 9:e54895.

24. Jia X, Burugula BB, Chen V, Lemons RM, Jayakody S, Maksutova M, et al. Massively parallel functional testing of MSH2 missense variants conferring Lynch syndrome risk. Am J Hum Genet. 2021, 108(1):163-175.

25. Shalem O, Sanjana NE, Hartenian E, Shi X, Scott DA, Mikkelson T, et al. Genome-scale CRISPR-Cas9 knockout screening in human cells. Science. 2014, 343(6166):84-87.

26. Bushnell B, Rood J, Singer E. BBMerge - Accurate paired shotgun read merging via overlap. PLoS One. 2017, 12(10):e0185056. 
27. Edgar RC. Search and clustering orders of magnitude faster than BLAST. Bioinformatics 2010, 26(19):2460-2461.

28. Buchfink B, Xie C, Huson DH. Fast and sensitive protein alignment using DIAMOND. Nat Methods. 2015, 12(1):59-60.

29. Graham RP, Treece AL, Lindeman NI, Vasalos P, Shan M, Jennings LJ, et al. Worldwide Frequency of Commonly Detected EGFR Mutations. Arch Pathol Lab Med. 2018, 142(2):163-167.

30. Yoshikawa K, Hamada J, Tada M, Kameyama T, Nakagawa K, Suzuki Y, et al. Mutant p53 R248Q but not R248W enhances in vitro invasiveness of human lung cancer $\mathrm{NCl}-\mathrm{H} 1299$ cells. Biomed Res. 2010, 31(6):401-411.

31. Okamoto K, Okamoto I, Okamoto W, Tanaka K, Takezawa K, Kuwata K, et al. Role of survivin in EGFR inhibitor-induced apoptosis in non-small cell lung cancers positive for EGFR mutations. Cancer Res. 2010, 70(24):10402-10410.

32. Wind S, Schmid M, Erhardt J, Goeldner RG, Stopfer P. Pharmacokinetics of afatinib, a selective irreversible ErbB family blocker, in patients with advanced solid tumours. Clin Pharmacokinet. 2013, 52(12):1101-1109.

33. Arulananda S, Do H, Musafer A, Mitchell P, Dobrovic A, John T. Combination Osimertinib and Gefitinib in C797S and T790M EGFR-Mutated Non-Small Cell Lung Cancer. J Thorac Oncol. 2017, 12(11):1728-1732.

34. Wang Z, Yang JJ, Huang J, Ye JY, Zhang XC, Tu HY, et al. Lung Adenocarcinoma Harboring EGFR T790M and In Trans C797S Responds to Combination Therapy of First- and Third-Generation EGFR TKIs and Shifts Allelic Configuration at Resistance. J Thorac Oncol. 2017, 12(11):1723-1727.

35. Hidaka N, Iwama E, Kubo N, Harada T, Miyawaki K, Tanaka K, et al. Most T790M mutations are present on the same EGFR allele as activating mutations in patients with non-small cell lung cancer. Lung Cancer. 2017, 108:75-82.

36. Brown BP, Zhang YK, Westover D, Yan Y, Qiao H, Huang V, et al. On-target Resistance to the MutantSelective EGFR Inhibitor Osimertinib Can Develop in an Allele-Specific Manner Dependent on the Original EGFR-Activating Mutation. Clin Cancer Res. 2019, 25(11):3341-3351.

37. Qin $Y$, Jian $H$, Tong $X$, Wu X, Wang F, Shao YW, et al. Variability of EGFR exon 20 insertions in 24468 Chinese lung cancer patients and their divergent responses to EGFR inhibitors. Mol Oncol. 2020, 14(8):1695-1704.

38. Hayashi $H$, lihara $H$, Hirose $C$, Fukuda $Y$, Kitahora $M$, Kaito D, et al. Effects of pharmacokineticsrelated genetic polymorphisms on the side effect profile of afatinib in patients with non-small cell lung cancer. Lung Cancer. 2019, 134:1-6.

39. Brown K, Comisar C, Witjes H, Maringwa J, de Greef R, Vishwanathan K, et al. Population pharmacokinetics and exposure-response of osimertinib in patients with non-small cell lung cancer. Br J Clin Pharmacol. 2017, 83(6):1216-1226.

40. Zofia Piotrowska YW, Lecia V. Sequist, Suresh S. Ramalingam. ECOG-ACRIN 5162: A phase II study of osimertinib 160 mg in NSCLC with EGFR exon 20 insertions. Journal of Clinical Oncology. 2020, 
38 suppl 15:9513.

41. Vyse S, Huang PH. Targeting EGFR exon 20 insertion mutations in non-small cell lung cancer. Signal Transduct Target Ther. 2019, 4:5.

42. Roskoski R, Jr. Properties of FDA-approved small molecule protein kinase inhibitors: A 2020 update. Pharmacol Res. 2020, 152:104609.

43. Sun $W$, Yuan X, Tian Y, Wu H, Xu H, Hu G, et al. Non-invasive approaches to monitor EGFR-TKI treatment in non-small-cell lung cancer. J Hematol Oncol. 2015, 8:95.

44. Pines G, Fankhauser RG, Eckert CA. Predicting Drug Resistance Using Deep Mutational Scanning. Molecules. 2020, 25(9):2265.

45. Bulbul A, Leal A, Husain H. Applications of cell-free circulating tumor DNA detection in EGFR mutant lung cancer. J Thorac Dis. 2020, 12(5):2877-2882.

46. Zhou Q, Yang JJ, Chen ZH, Zhang XC, Yan HH, Xu CR, et al. Serial cfDNA assessment of response and resistance to EGFR-TKI for patients with EGFR-L858R mutant lung cancer from a prospective clinical trial. J Hematol Oncol. 2016, 9(1):86.

47. Wang S, Tsui ST, Liu C, Song Y, Liu D. EGFR C797S mutation mediates resistance to third-generation inhibitors in T790M-positive non-small cell lung cancer. J Hematol Oncol. 2016, 9(1):59.

\section{Figures}




\section{Figure 1}

\section{Construction of libraries}

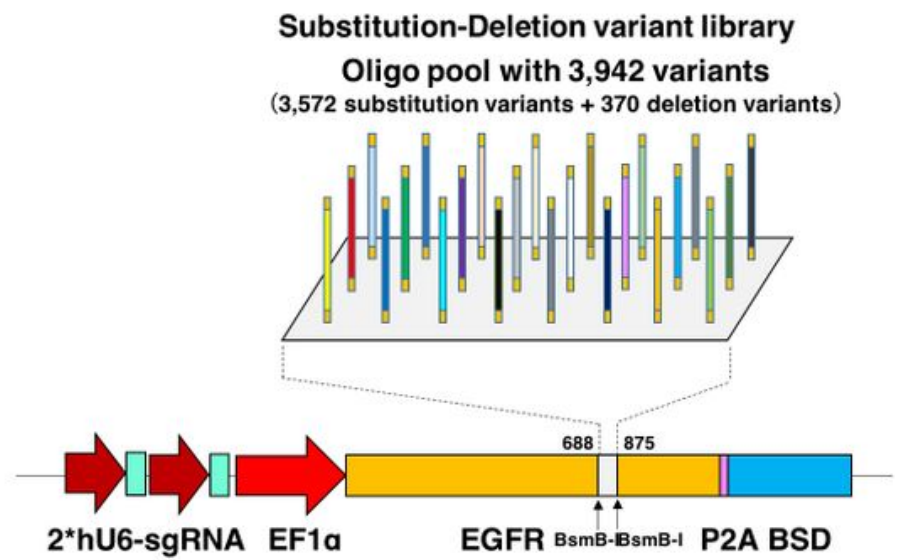

2. Lentivirus packaging
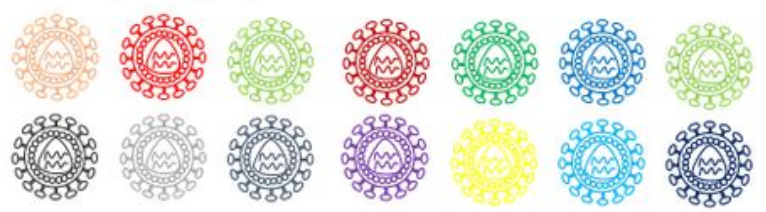

Insertion variant library

Degenerate primers with 1, 2 or 3 NNS codons inserted (NNS) (NNSNNS) (NNSNNSNNS) (300 variants) $\quad(6,000$ variants $) \quad(120,000$ variants $)$

(6,000 variants) (120,000 variants)
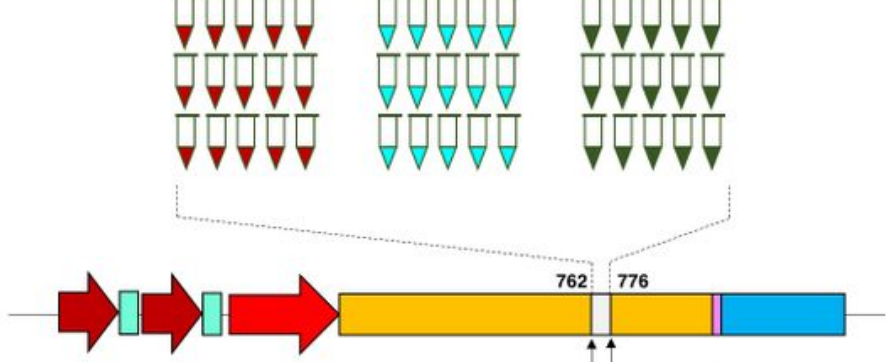

2*hU6-sgRNA EF1a

4. Tyrosine kinase inhibitor assays

Time 0

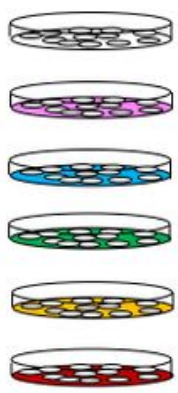

Cell counting (Once every two days)

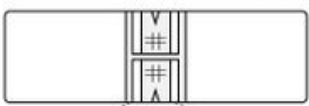

Afatinib: $50 \mathrm{nM} / 600 \mathrm{nM}$

Erlotinib: $600 \mathrm{nM}$

Gefitinib: $600 \mathrm{nM}$

Icotinib: $600 \mathrm{nM}$

Osimertinib: $200 \mathrm{nM} 600 \mathrm{nM}$

3. Virus transduction

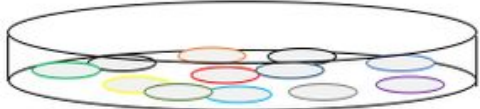

5. High-throughput sequencing

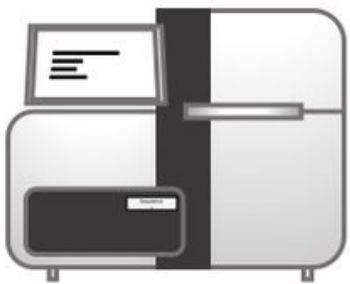

6. Data analysis

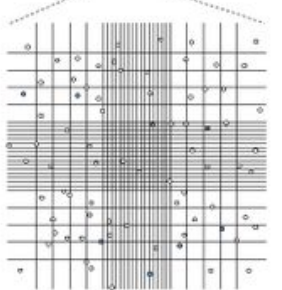

Time 1

(Two weeks later)

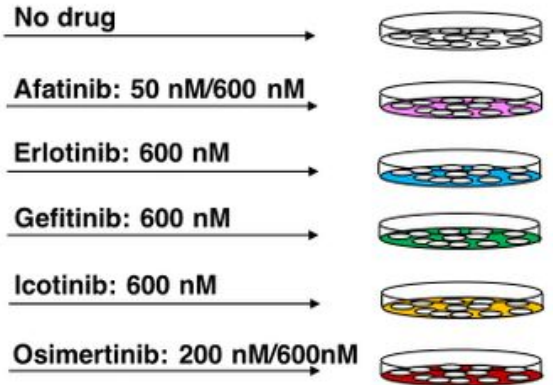

\begin{tabular}{|l|l|l|l|}
\hline Variant & Cell number (Pre) & Cell number (Post) & Enrichment Score \\
\hline 1 & $\mathrm{n} 1$ & $\mathrm{n} 1^{\prime}$ & $\mathrm{n} 1^{\prime} / \mathrm{n} 1$ \\
\hline 2 & $\mathrm{n} 2$ & $\mathrm{n} 2^{\prime}$ & $\mathrm{n} 2^{\prime} / \mathrm{n} 2$ \\
\hline$\cdots$ & & & \\
\hline $\mathrm{N}$ & & & \\
\hline
\end{tabular}

Figure 1

Overview of the experimental design and data analysis. Parallelly synthesized oligonucleotide pools and degenerate primers were separately used to construct EGFR substitution-deletion (Sub-Del) and insertion (Ins) variant libraries. Variants in the Sub-Del library were located within EGFR exons 18-21 (amino acid positions, 688-875). Variants in the Ins library were located within EGFR exon 20 (amino acid positions, 762-776). Each variant had only one designed mutation within a full codon-optimized EGFR-coding 
sequence. Two single-guide RNA (sgRNA) expression cassettes driven by human U6 (hU6) promoter, targeting the endogenous EGFR sequence, were located upstream of the EGFR variant expression module. A blasticidin S resistance gene (BSD) was fused with the EGFR variant cassette through a P2A peptide. The Sub-Del and Ins libraries were separately introduced into Cas9-expressing PC9 cells. The cells were treated with five EGFR tyrosine kinase inhibitors (TKIs) or dimethyl sulfoxide (DMSO). The number of cells in each assay was counted manually once, every 2 days. Two weeks later, cells were harvested and subjected to genomic DNA extraction. Mutation regions were amplified by polymerase chain reaction and subjected to next-generation sequencing. Relative drug sensitivity was determined by variant enrichment in cell numbers before and after drug screening. 
Figure 2

a

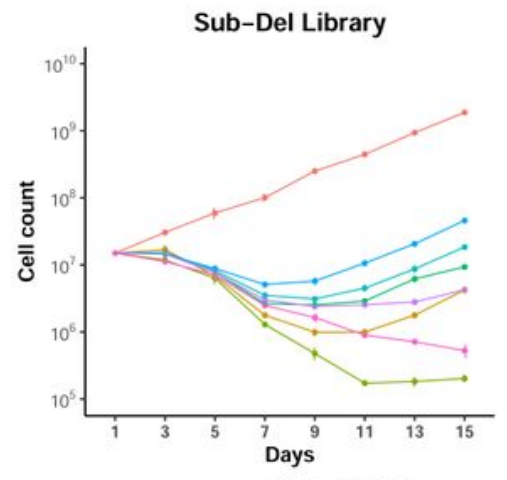

C

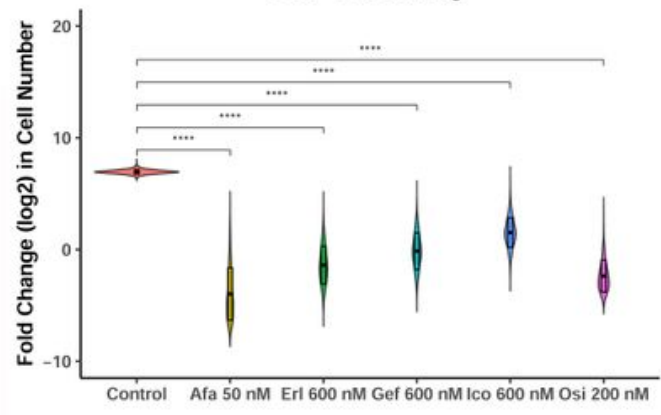

b
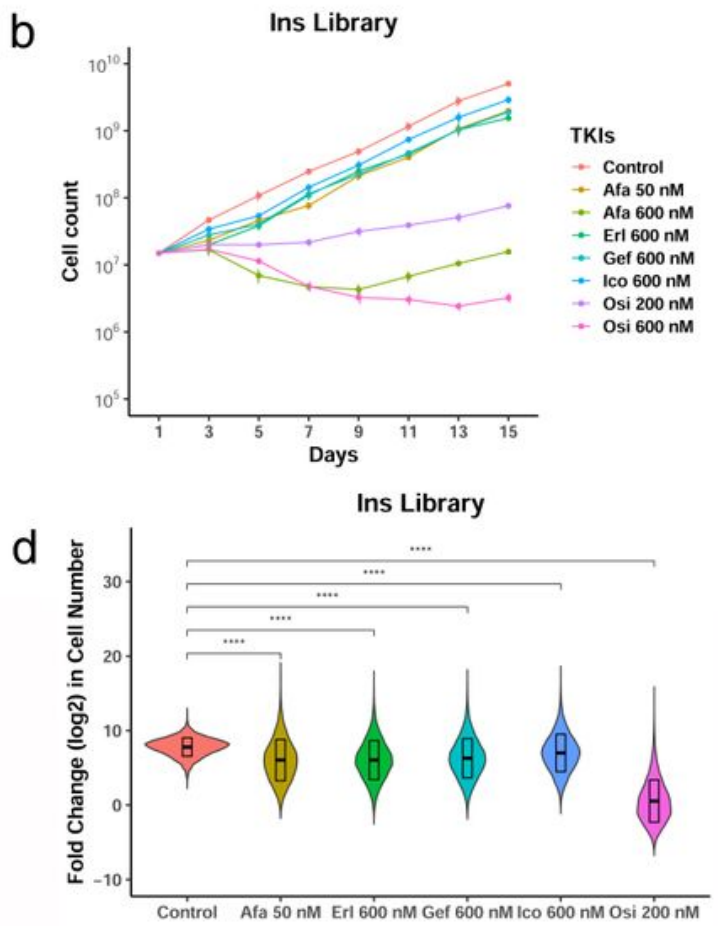

e
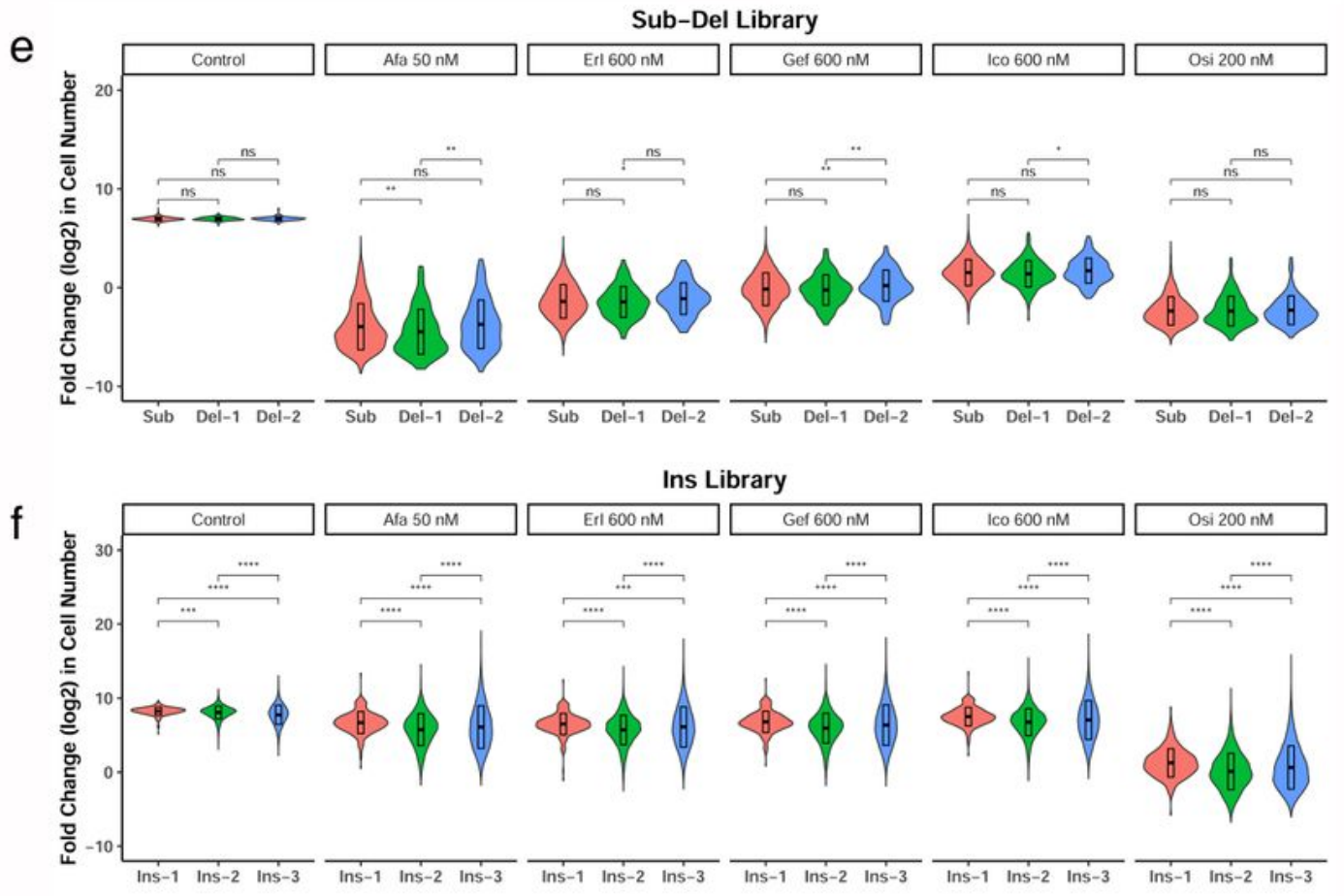

\section{Figure 2}

EGFR-tyrosine kinase inhibitors (EGFR-TKIs) showed different inhibition on Sub-Del and Ins library variants. Cell growth kinetics $(\log 10)$ of the Sub-Del $(a)$ and Ins (b) libraries treated with five TKIs and dimethyl sulfoxide (DMSO) (mechanical replicates, $n=4$; error bars indicate the standard deviation). Violin plots denoting enrichment score ( $\log 2)$ distributions of variants in the Sub-Del (c) and Ins (d) libraries treated with the five TKIs or DMSO. e The enrichment scores $(\log 2)$ of the three subtypes of 
variants (Sub: single amino acid (AA) substitution; Del-1: single AA deletion; and Del-2: dual AA deletion) in the Sub-Del library are separately displayed by treatment type in the violin plot. $f$ The enrichment scores (log2) of the three subtypes of variants (Ins-1: single AA insertion; Ins-2: dual AA insertion; and Ins-3: triple AA insertion) in the Ins library are separately displayed by treatment type in the violin plot. Student's t-test, ns: $P>0.05, *: P \leq 0.05, * *: P \leq 0.01, * * *: P \leq 0.001, * \star * *: P \leq 0.0001$.

Figure 3

a

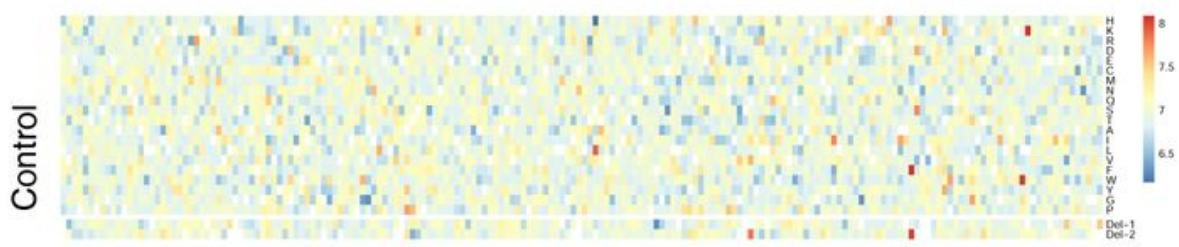

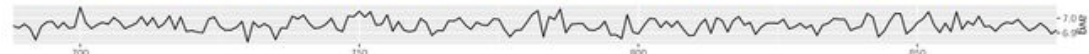

b

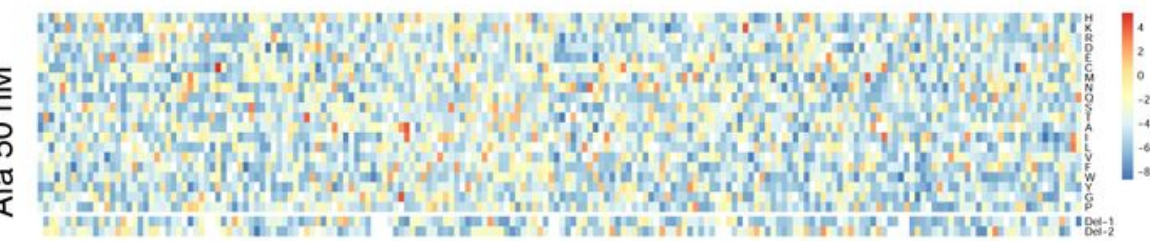

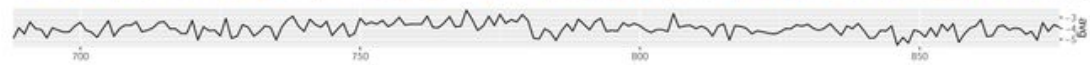

C

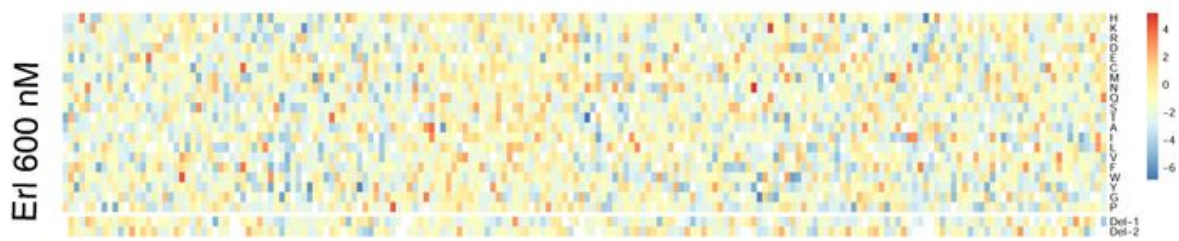

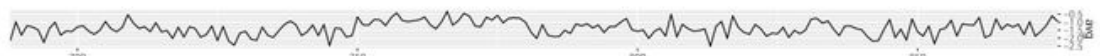

d

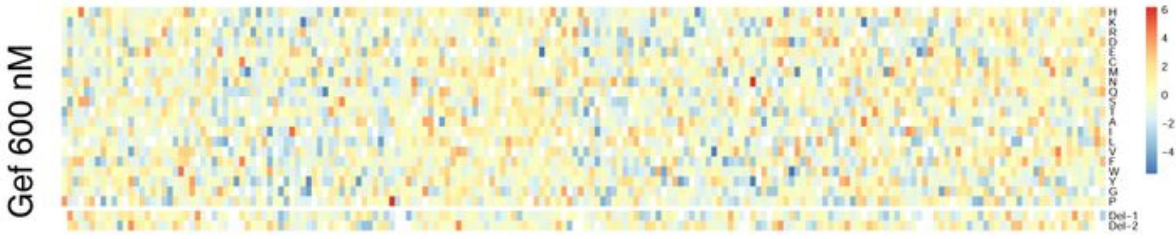

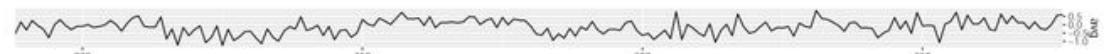

e
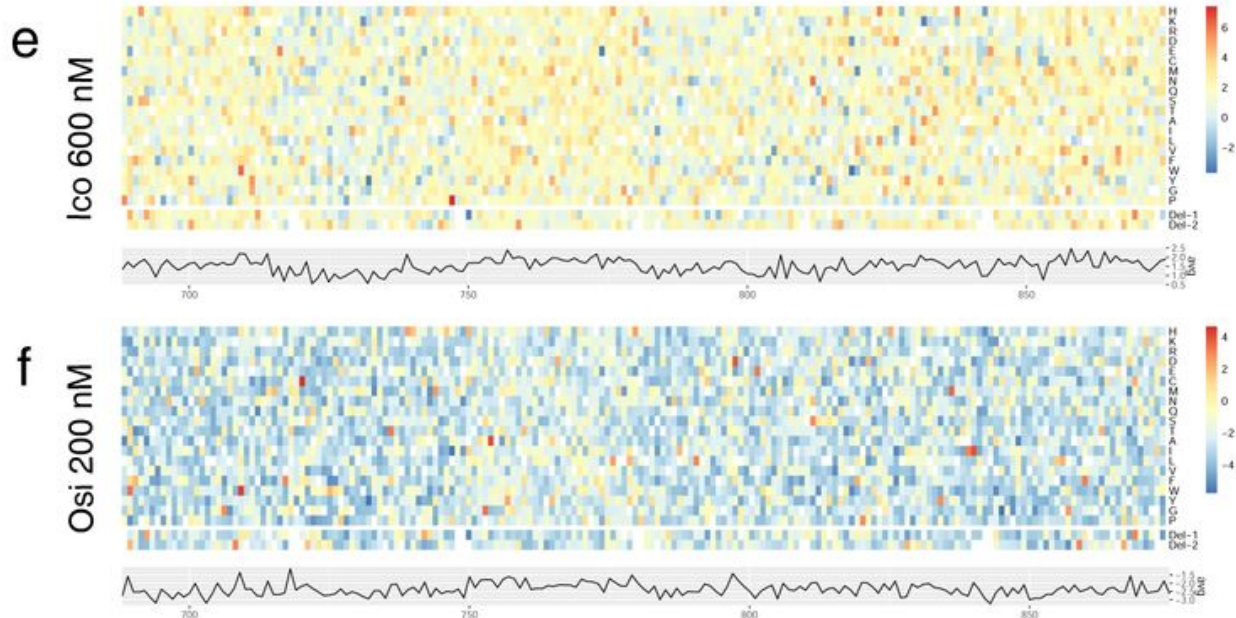

Figure 3 
Heatmaps depicting enrichment of EGFR Sub-Del library variants on the cytotoxicity of five tyrosine kinase inhibitors (TKIs). The enrichment scores are shown as the ratio (log2) of the cell number post-TKI treatment to that pre-TKI treatment. The top 20 rows represent all 20 possible amino acid (AA) substitutions, and the two rows below represent one or two AA deletion mutations (Sub: single AA substitution; Del-1: single AA deletion; and Del-2: dual AA deletion). Each column indicates one AA residue in EGFR (688-875). Blue to red represents high to low abundance of variants, respectively. Variants that were not covered are colored in white. Codon-level average enrichment scores are plotted below each heatmap. Cells were treated with dimethyl sulfoxide (DMSO) (a), $50 \mathrm{nM}$ afatinib (b), $600 \mathrm{nM}$ erlotinib (c), $600 \mathrm{nM}$ gefitinib (d), $600 \mathrm{nM}$ icotinib (e), or $200 \mathrm{nM}$ osimertinib (f).

\section{Figure 4}

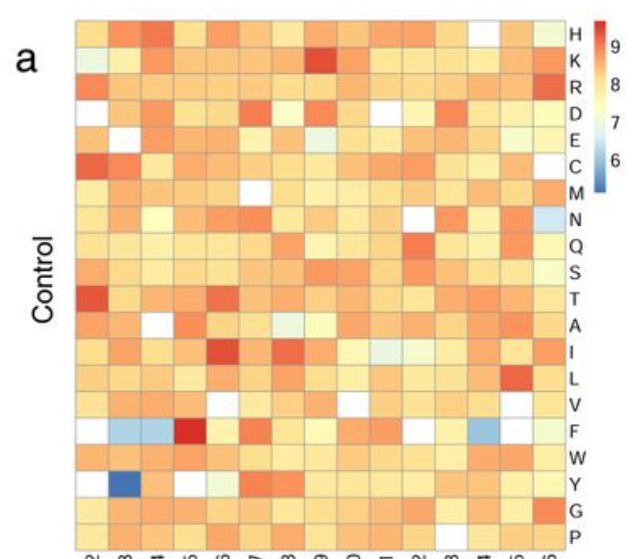

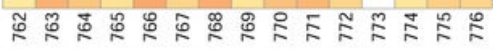

$\begin{array}{llllllll}7 \dot{6} 2 & 7 \dot{6} 4 & 7 \dot{6} 6 & 7 \dot{6} 8 & 770 & 772 & 7 \dot{7} 4 & 7 \dot{7} 6\end{array}$

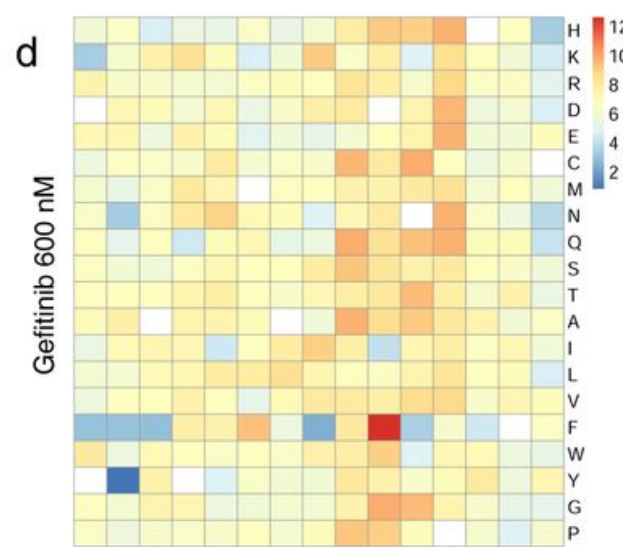

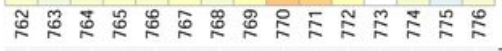

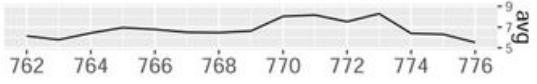

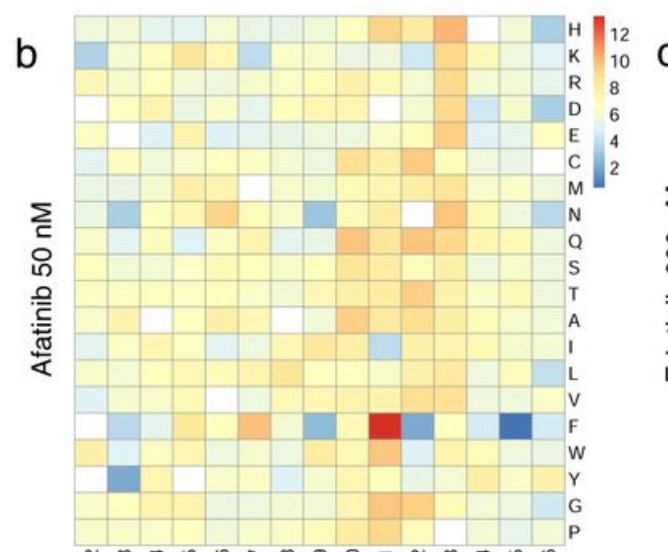

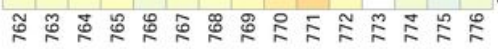
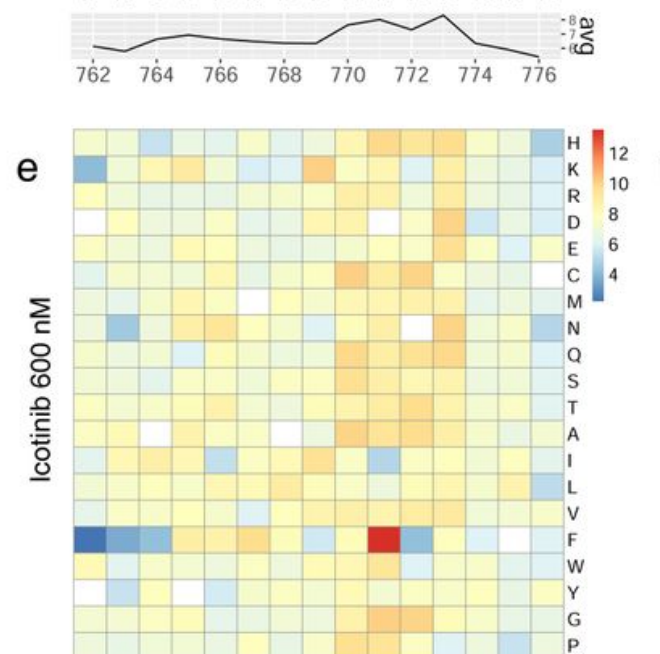

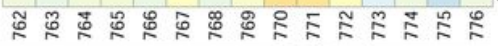

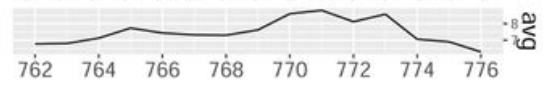

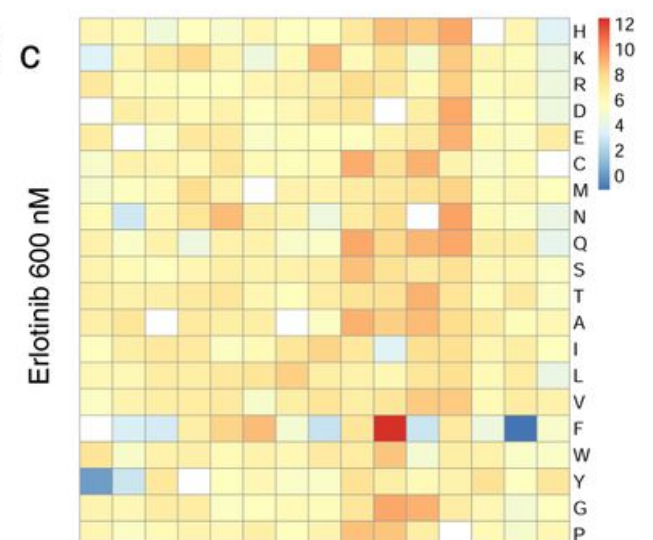

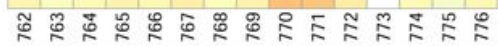
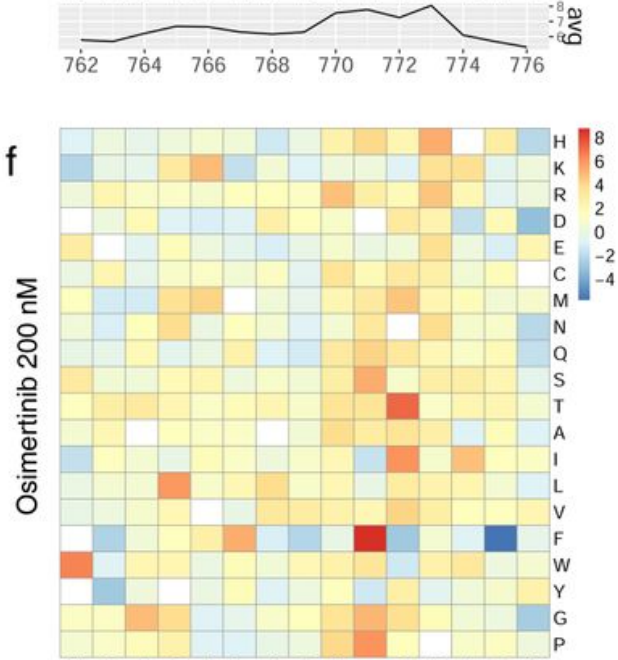

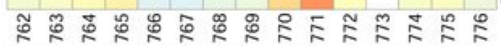

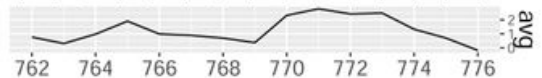

\section{Figure 4}

Heatmaps depicting the enrichment results for EGFR Ins-1 library variants on the cytotoxicity of five tyrosine kinase inhibitors (TKIs). The enrichment scores are shown as the ratio (log2) of the cell number post-TKI treatment to that pre-TKI treatment. The 20 rows represent all 20 possible single AA insertion mutations. Each column indicates one AA residue in EGFR (762-776). Blue to red represents high to low abundance of variants, respectively. Variants that were not covered are colored in white. Codon-level 
average enrichment scores are plotted below each heatmap. Cells were treated with dimethyl sulfoxide (DMSO) (a), $50 \mathrm{nM}$ afatinib (b), $600 \mathrm{nM}$ erlotinib (c), $600 \mathrm{nM}$ gefitinib (d), $600 \mathrm{nM}$ icotinib (e), or $200 \mathrm{nM}$ osimertinib (f).

\section{Figure 5}

a

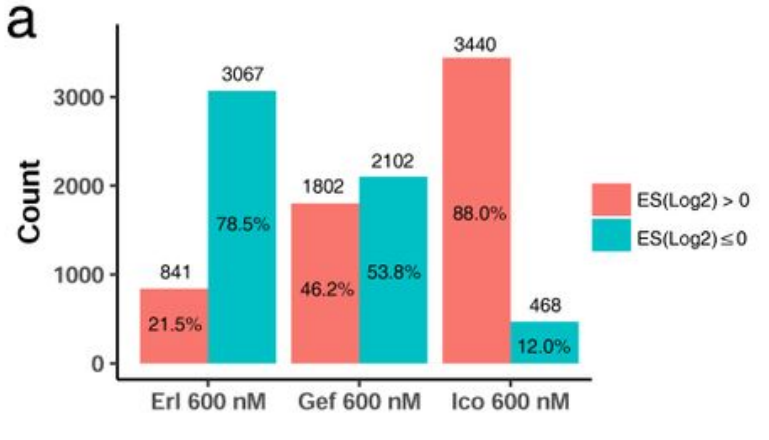

C

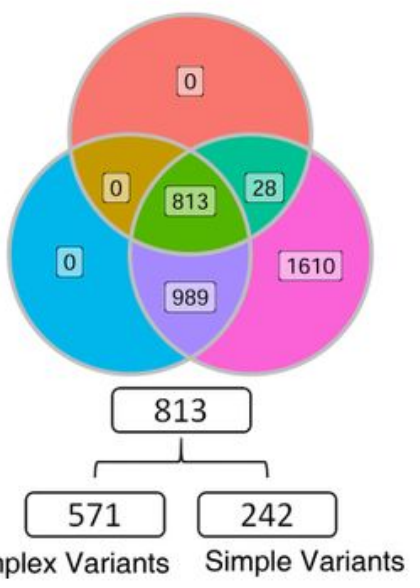

e

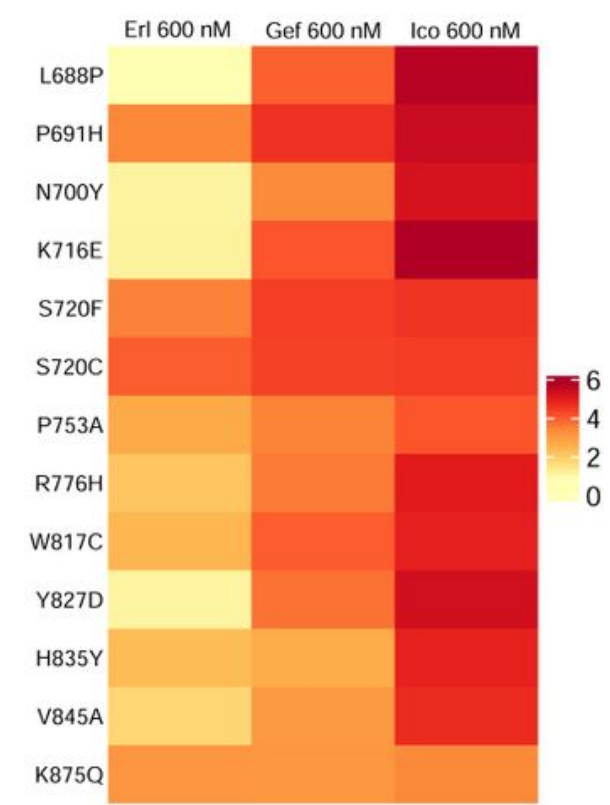

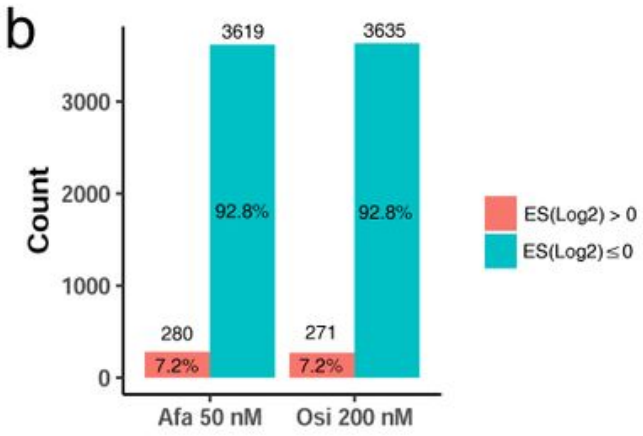

d
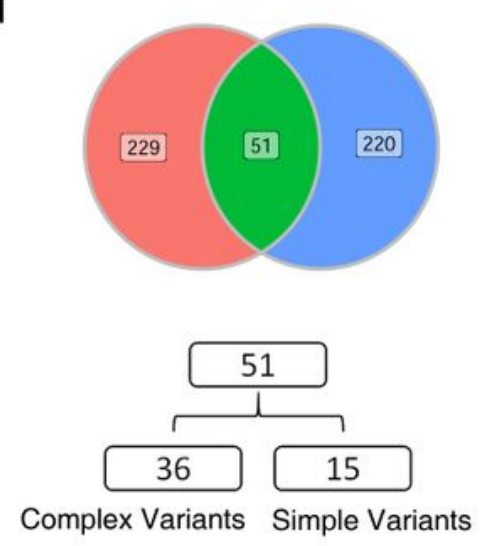

f

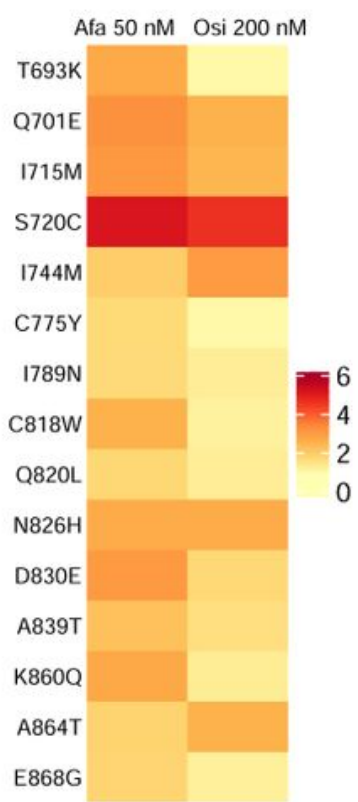

\section{Figure 5}

Potentially drug-resistant substitutions identified in the cytotoxicity screening. Number and proportion of enriched (red bar) and depleted (green bar) variants in the reversible (a) and irreversible (b) tyrosine 
kinase inhibitor (TKI) assays. c The enriched variants in the three reversible TKI assays have an intersection of 813 variants (571 complex and 242 simple variants). $d$ The enriched variants in the two irreversible TKI assays have an intersection of 51 variants (36 complex and 15 simple variants). e The enrichment score ( $\log 2)$ of the top $5 \%$ of the selected simple variants in "c" is presented as a heatmap. $f$ The enrichment score $(\log 2)$ of all the 15 selected simple variants in "d" was presented as a heatmap. Afa, Erl, Gef, Ico, Osi, and ES are abbreviations for afatinib, erlotinib, gefitinib, icotinib, osimertinib, and enrichment score, respectively.

Figure 6

a Afatinib (50 $\mathrm{nM})$

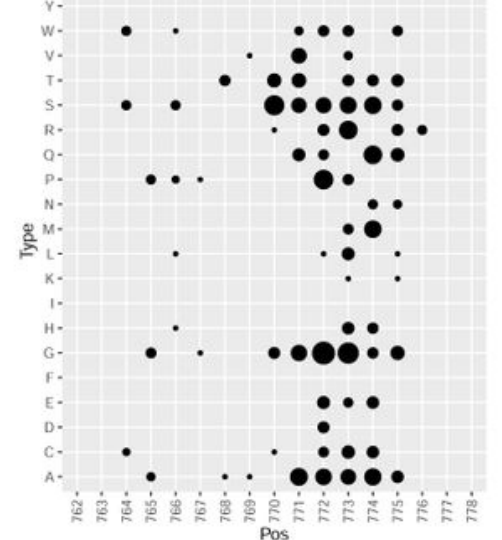

d Icotinib $(600 \mathrm{nM})$

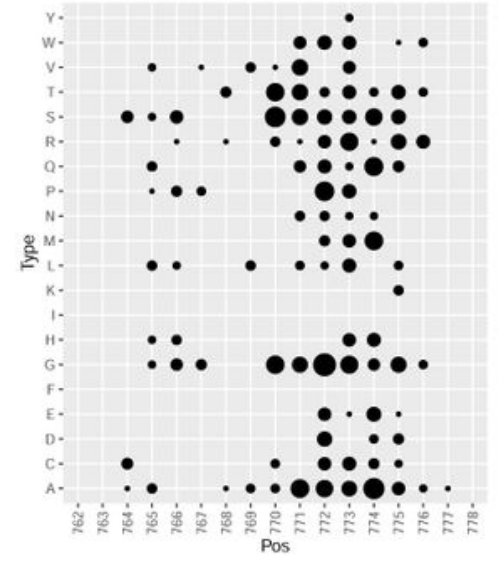

g

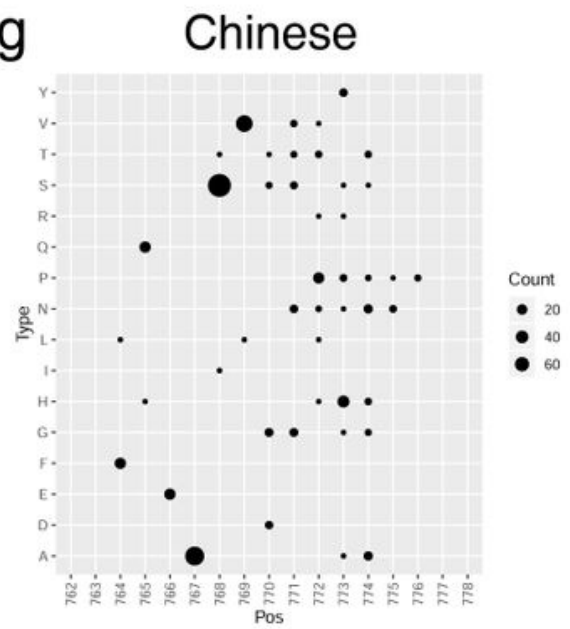

b Erlotinib (600 $\mathrm{nM})$

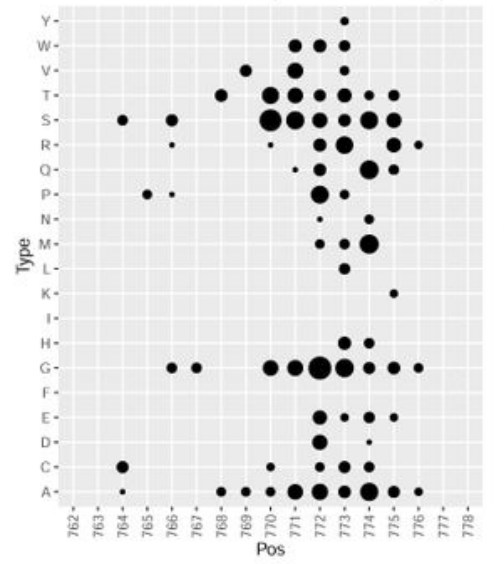

e Osimertinib (200 nM)

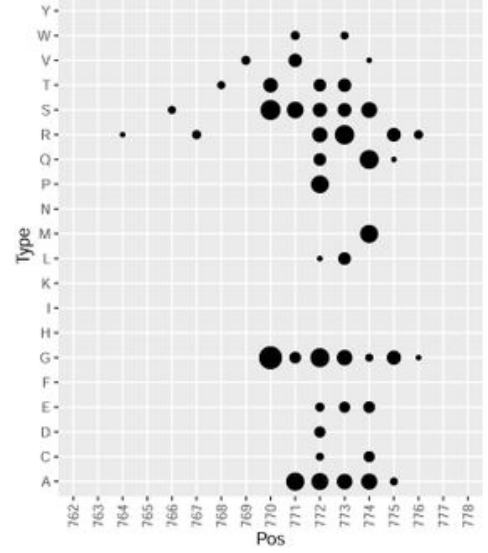

$\mathrm{h}$
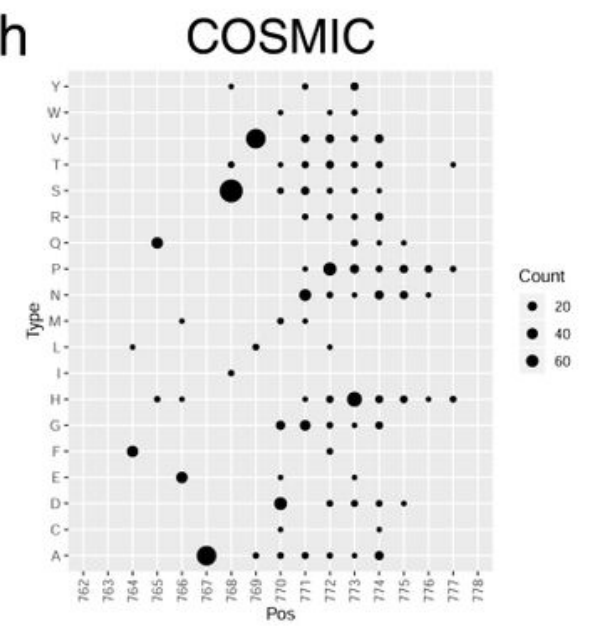

C Gefitinib (600 nM)
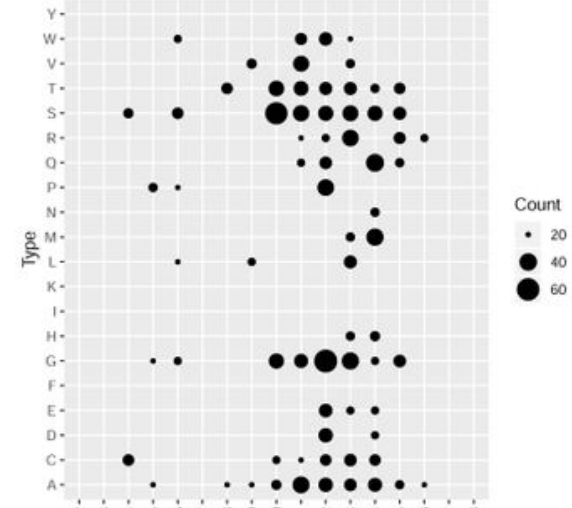

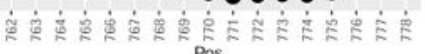

Control

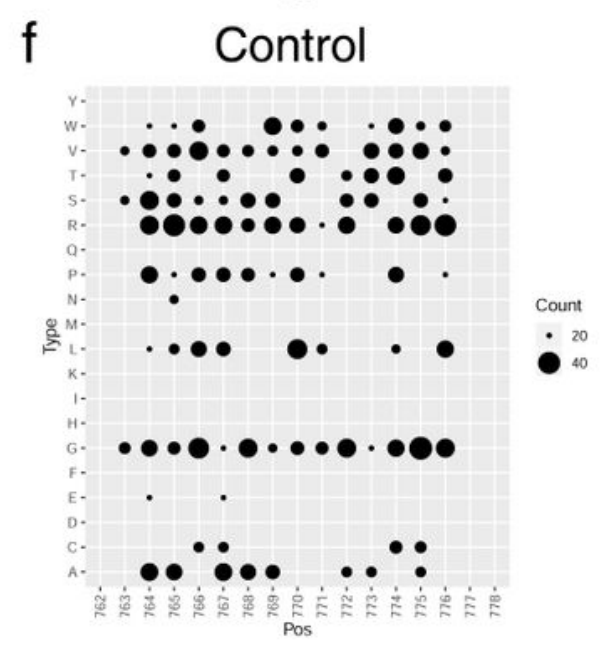

Combined
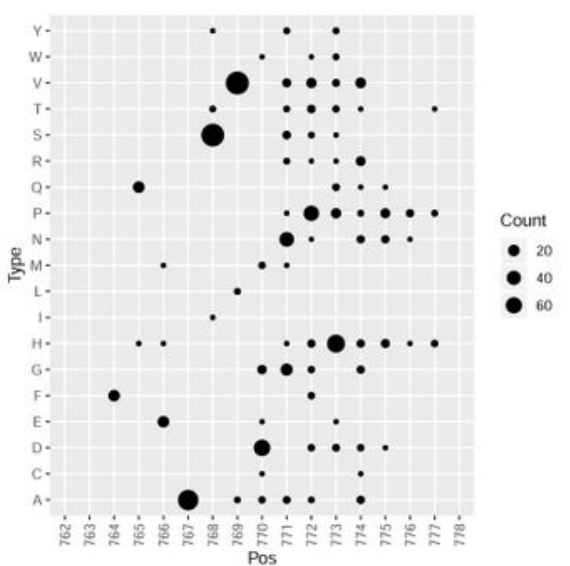


\section{Figure 6}

Variants in EGFR exon 20 insertional hotspot induce stronger tyrosine kinase inhibitor (TKI) resistance. a-f The inserted amino acids (AA) in the top 5\% enriched exon 20 insertions were counted and the cumulative frequency numbers were plotted as black dots at the corresponding AA positions. Dots with cumulative frequency less than 15 were filtered. a-e show summary results for afatinib (50 nM), erlotinib, gefitinib, icotinib, and osimertinib (200 nM) assays, respectively. f shows the summary result for untreated control. $g-i$ The inserted AA in exon 20 insertions from the Chinese population [37] and COSMIC database were counted and the cumulative frequency numbers were plotted as black dots at the corresponding $A A$ positions. All the dots are shown, regardless of the cumulative frequency numbers. $g$ shows the summary result for the Chinese population. $\mathrm{h}$ shows the summary result for the COSMIC database. i shows combined results of " $\mathrm{g}$ " and " $\mathrm{h}$ ". The $\mathrm{X}$-axis shows AA positions.

\section{Supplementary Files}

This is a list of supplementary files associated with this preprint. Click to download.

- Additionalfile1FigureS1.docx

- Additionalfile2FigureS2.docx

- Additionalfile3FigureS3.docx

- Additionalfile4FigureS4.docx

- Additionalfile5FigureS5.docx

- Additionalfile6FigureS6.docx

- Additionalfile7FigureS7.docx

- Additionalfile8FigureS8.docx

- Additionalfile9FigureS9.docx

- Additionalfile10Tables1.docx

- Additionalfile11Tables2.docx

- Additionalfile12Tables3.docx 